\title{
Fourier Series Expansions of Powers of the Trigonometric Sine and Cosine Functions
}

\author{
M.A. Sharaf ${ }^{1}$, M.S. Algorabi ${ }^{2}$ \\ ${ }^{1}$ Department of Astronomy, Faculty of Science, King Abdulaziz University, Jeddah, Saudi Arabia \\ Sharaf_adel@hotmail.com \\ ${ }^{2}$ Department of Mathematics, Batterjee Education \& Training Academy, Jeddah, Saudi Arabia \\ Maha-algorabi@hotmail.com
}

\begin{abstract}
In this paper, Fourier series expansions of powers of sine and cosine functions are established for any possible powerreal or complex or positive integer. Recurrence relations are established to facilities the computations of the coefficients of expansions formulae. Numerical applications for real and complex powers are also included, the accuracy of the computed values are at least of order $10^{-10}$. While the applications for positive integer powers are given as exact analytical expressions.
\end{abstract}

\section{Indexing terms/Keywords}

Fourier series expansions; fraction and complex powers of sine and cosine; recursive algorithms.

\section{Academic Discipline And Sub-Disciplines}

Celestial Mechanics; Dynamical Astronomy.

\section{SUBJECT CLASSIFICATION}

Celestial Mechanics.

\section{TYPE (METHOD/APPROACH)}

Fourier series expansions of powers of the trigonometric sine and cosine functions powers of sine and cosine functions.

\section{INTRODUCTION}

The wide reneges of differentiability, continuity and integralability of the sine and cosine functions, make the Fourier series expansions (FSE) are the most powerful tools for representing periodic functions as sums of these functions. Consequently, (FSE) are the suitable expansions for solving certain classical problems of applied mathematics of these are for examples, the higher order partial differential equations, electromagnetic theory, wave kinematics, rotor-seal systems etc.

On the other hand, the powers of sine and cosine functions appeared in many problems of space dynamics [e.g. in the series of papers by Sharaf, M.A. entitled as "Expansion theory for the elliptic motion of arbitrary eccentricity and semi major axis .Published in Astrophysics and Space Science .since 1981-1986"]., also it appeared in analysis of light curves of eclipsing variable stars.

Due the importance of the (FSE) as mentioned in brief as to in the above, the present paper is devoted to establish, (FSE) of powers of sine and cosine for any possible power-real or complex or positive integer. Recurrence relations are established to facilities the computations of the coefficients of expansions formulae.

Numerical applications for real and complex powers are also included, the accuracy of the computed values are at least of order $10^{-10}$. While the applications for positive integer powers are given as exact analytical expressions.

\section{FOURIER EXPANSIONS OF $\sin ^{2 x} x \& \cos ^{2 v} x$}

\subsection{Formulations}

In this section the (FSE) for any real or complex $v$ such that $\operatorname{Re} v \succ-\frac{1}{2}$ (the case of positive integers will be considered in Section 3 ) will be established for $\sin ^{2 v} x \quad \forall 0 \prec x \prec \pi$, and for $\cos ^{2 v} x \forall \frac{-\pi}{2} \prec x \prec \frac{\pi}{2}$. Since

$$
\begin{aligned}
& \sin ^{p} x=\frac{(-J)^{p}}{2^{p}}\left(\Phi-\Phi^{-1}\right)^{p} \\
& \cos ^{q} x=\frac{1}{2^{q}}\left(\Phi+\Phi^{-1}\right)^{q}
\end{aligned}
$$


where

$$
\mathrm{J}=\sqrt{-1} ; \Phi=\exp (\mathrm{Jx})
$$

Using the binomial theorem, we deduce that

$$
\begin{gathered}
\sin ^{2 v} x=\frac{1}{2} a_{0}+\sum_{n=1}^{\infty} a_{n} \cos 2 n x ; \operatorname{Re} v \succ-\frac{1}{2} ; 0 \prec x \prec \pi, \\
\cos ^{2 v} x=\frac{1}{2} a_{0}+\sum_{n=1}^{\infty}(-1)^{n} a_{n} \cos 2 n x ; \operatorname{Re} v \succ-\frac{1}{2} ;-\frac{\pi}{2} \prec x \prec \frac{\pi}{2},
\end{gathered}
$$

where

$$
a_{n}=\frac{(-1)^{n} I(1+2 v)}{2^{2 v} I(1+v+n) I(1+v-n)} ; n \geq 0
$$

and $\Gamma$ is the Gamma function.

\subsection{Computational developments}

\subsubsection{Recurrence relations for a's coefficients}

- For real $v$

Since $I(x+1)=x I(x)$, then we get from Equation (3), the recurrence relation

$$
a_{n+1}=\frac{n-v}{1+n+v} a_{n} ; n \geq 0
$$

where

$$
a_{0}=\frac{I(1+2 v)}{2^{2 v-1}(I(1+v))^{2}}
$$

- For complex $v$

Let

$$
v=v_{1}+J v_{2} ; J=\sqrt{-1}
$$

So, the coefficients becomes complex in he form

$$
a_{n}=q_{n}+J h_{n} .
$$

Consequently, Equation(4.1) becomes

$$
q_{n+1}+J h_{n+1}=\frac{n-v_{1}-J v_{2}}{1+n+v_{1}+J v_{2}}\left(q_{n}+J h_{n}\right) ; n \geq 0
$$

Equating the real and imaginary parts of this equation we get the recurrence relations

$$
\begin{aligned}
& q_{n+1}=\frac{\left(n-v_{1}\right)\left(1+n+v_{1}\right)-v_{2}^{2}}{\left(1+n+v_{1}\right)^{2}+v_{2}^{2}} q_{n}+\frac{\left.v_{6} 1+2 n\right)}{\left(1+n+v_{1}\right)^{2}+v_{2}^{2}} h_{n} ; n \geq 0, \\
& h_{n+1}=\frac{\left(n-v_{1}\right)\left(1+n+v_{1}\right)-v_{2}^{2}}{\left(1+n+v_{1}\right)^{2}+v_{2}^{2}} h_{n}+\frac{v(1+2 n)}{\left(1+n+v_{1}\right)^{2}+v_{2}^{2}} q_{n} ; n \geq 0 .
\end{aligned}
$$

where,

$$
\mathrm{q}_{0}=\operatorname{Re}\left[\frac{\mathrm{I}\left(1+2 v_{1}+\mathrm{J} 2 v_{2}\right)}{2^{2 v_{1}-1+2 J v_{2}}\left(\mathrm{I}\left(1+v_{1}+J v_{2}\right)\right)^{2}}\right] ; h_{0}=\operatorname{Im}\left[\frac{\mathrm{I}\left(1+2 v_{1}+J 2 v_{2}\right)}{2^{2 v_{1}-1+2 J v_{2}}\left(\mathrm{I}\left(1+v_{1}+J v_{2}\right)\right)^{2}}\right]
$$




\subsubsection{Accuracy checks}

The accuracy of the computed values may be checked by the conditions that:

$$
\begin{gathered}
\varepsilon=\sin ^{2}(x)-\left(\frac{1}{2} a_{0}+\sum_{n=1} a_{n} \cos 2 n x\right)_{\forall \in x] 0, \pi[}, \\
\left.\left.\varepsilon_{1}=\cos ^{2} \gamma x\right)-\left(\frac{1}{2} a_{0}+\sum_{n=1}-1\right)^{n} a_{n} \cos 2 n x\right)_{\forall x \in]-\pi / 2, \pi / 2[},
\end{gathered}
$$

where $\varepsilon$ and $\varepsilon_{1}$ are small tolerances of $\approx \emptyset 10^{-10}$ ) at least.

\subsubsection{Numerical examples}

\section{-Example 1}

Consider $v=5.94$, with this value of $v$ we get for the coefficients of (FSE) of $\sin ^{2 v} x$ the first twenty values which are listed in columns $2 \& 6$ from table 1 , where $a_{0}=0.453349713$. The values of angle $x$ are listed in columns $3 \& 7$, while, the accuracy cheeks $\varepsilon$ are given in columns $4 \& 8$.

\section{-Example 2}

The corresponding computations for $\cos ^{2 v} \mathrm{x}$ are listed in Table 2, where $v=3.765$ and $\mathrm{a}_{0}=0.562594$.

\section{EExample 3}

The results for Fourier expansion of $\sin ^{2 v} x$ with complex $v=2.67+2 \mathrm{i}$ and $\mathrm{a}_{0}=0.572892-0.176663 \mathrm{i}$ are listed in Table 3 .

\section{Example 4}

Finally, the results for Fourier expansion of $\cos ^{2 v} x$ with complex $v=3.765+2 i$ and $a_{0}=0.518449-0.121644 i$ are listed in Table 4.

\begin{tabular}{|c|c|c|c|c|c|c|c|}
\hline$i$ & $a_{i}$ & $\mathbf{x}^{0}$ & $\square$ & i & $a_{i}$ & $x^{0}$ & $\square$ \\
\hline 1 & 0.388026 & 1.61731 & $8.78464 \square 10^{14}$ & 11 & $9.82691 \square 10^{\square 10}$ & 51.9953 & $6.80289 \square 10^{14}$ \\
\hline 2 & 0.241416 & 87.4289 & $2.44527 \square 10^{14}$ & 12 & $2.77169 \square 10^{\square 10}$ & 10.5793 & $\square 7.18869 \square 10^{14}$ \\
\hline 3 & $\square 0.106396$ & 35.874 & $\square 1.86795 \square 10^{14}$ & 13 & $8.86824 \square 10^{\square 11}$ & 24.997 & $1.63869 \square 10^{\square 3}$ \\
\hline 4 & 0.0314693 & 38.3996 & $\square 1.14825 \square 10^{13}$ & 14 & $3.13991 \square 10^{11}$ & 164.199 & $1.84519 \square 10^{13}$ \\
\hline 5 & $\square 0.00558047$ & 161.314 & $\square 1.78468 \square 10^{14}$ & 15 & $1.20858 \square 10^{\square 11}$ & 28.1381 & $\square 1.06887 \square 10^{13}$ \\
\hline 6 & 0.000439334 & 37.2613 & $\square 1.16629 \square 10^{13}$ & 16 & $4.99076 \square 10^{112}$ & 32.1528 & $7.56062 \square 10^{14}$ \\
\hline 7 & $2.0371 \square 10^{6}$ & 80.7445 & $7.79654 \square 10^{14}$ & 17 & $2.18863 \square 10^{12}$ & 86.5704 & $6.71407 \square 10^{14}$ \\
\hline 8 & $1.54901 \square 10^{\square}$ & 89.0032 & $\square 6.22002 \square 10^{14}$ & 18 & $1.01112 \square 10^{\square 12}$ & 135.915 & $1.95399 \square 10^{14}$ \\
\hline 9 & $2.13585 \square 10^{8}$ & 160.248 & $\square 1.44357 \square 10^{13}$ & 19 & $4.88938 \square 10^{\square 13}$ & 32.1719 & $7.71883 \square 10^{14}$ \\
\hline 10 & $4.10019 \square 10^{9}$ & 144.703 & $3.60545 \square 10^{14}$ & 20 & $2.46165 \square 10^{\square 13}$ & 51.9078 & $7.28029 \square 10^{14}$ \\
\hline
\end{tabular}

Table 1. Fourier Expansion of $\sin ^{2 v} x$ and its Error Analysis

\begin{tabular}{|c|c|c|c|c|c|c|c|}
\hline 1 & 0.444526 & 88.3827 & $1.48426 \square 10^{10}$ & 11 & $\square .65227 \square 10^{8}$ & 38.0047 & $8.79626 \square 10^{11}$ \\
\hline 2 & 0.213203 & 2.57109 & $3.19526 \square 10^{11}$ & 12 & $2.59398 \square 10^{8}$ & 79.4207 & $\square 2.07767 \square 10^{10}$ \\
\hline 3 & 0.055625 & 54.126 & $\square 4.97047 \square 10^{11}$ & 13 & $\square 1.27417 \square 10^{8}$ & 65.003 & $2.43607 \square 10^{10}$ \\
\hline 4 & 0.00548012 & 51.6004 & $\square 1.5588 \square 10^{10}$ & 14 & $6.62367 \square 10^{9}$ & $\square 74.1991$ & $3.27874 \square 10^{10}$ \\
\hline 5 & $\square 0.000146928$ & $\square 71.3137$ & $\square 8.5577 \square 10^{11}$ & 15 & $\square 3.61275 \square 10^{9}$ & 61.8619 & $\square 1.82286 \square 10^{10}$ \\
\hline 6 & 0.0000185824 & 52.7387 & $\square 1.75916 \square 10^{10}$ & 16 & $2.05359 \square 10^{9}$ & 57.8472 & $1.33713 \square 10^{10}$ \\
\hline 7 & $\square 3.85802 \square 10^{6}$ & 9.25552 & $\square 1.09553 \square 10^{10}$ & 17 & $\square 1.21 \square 10^{\square}$ & 3.42959 & $9.08718 \square 10^{11}$ \\
\hline 8 & $1.06083 \square 10^{6}$ & 0.996754 & $\square 8.76215 \square 10^{11}$ & 18 & $7.35786 \square 10^{10}$ & $\square 45.9153$ & $1.24942 \square 10^{11}$ \\
\hline 9 & $\square 3.51949 \square 10 \square$ & $\square 70.2482$ & $\square 2.5846 \square 10^{10}$ & 19 & $\square 4.60089 \square 10^{10}$ & 57.8281 & $1.35819 \square 10^{10}$ \\
\hline 10 & $1.3385 \square 10^{7}$ & $\square 54.7029$ & $2.92011 \square 10^{11}$ & 20 & $2.94949 \square 10^{10}$ & 38.0922 & $9.51788 \square 10^{11}$ \\
\hline
\end{tabular}

Table 2. Fourier Expansion of $\cos ^{2 v} X$ and its Error Analysis 
Table 3. Fourier Expansion of $\sin ^{2 v} x$ with complex $v=2.67+2 i$ and its Error Analysis

\begin{tabular}{cccc}
$i$ & $a_{i}$ & $x^{0}$ & \\
\hline 1 & $\square 0.47276 \square 0.0739583 \square$ & 1.61731 & $3.34711 \square 10^{10} \square 5.36575 \square 10^{10} \square$ \\
2 & $0.233323 \square 0.0760953 \square$ & 87.4289 & $6.31528 \square 10^{12} \square 2.28166 \square 10^{11} \square$ \\
3 & $\square 0.0292874 \square 0.080962 \square$ & 35.874 & $\square 2.82208 \square 10^{12} \square 5.69501 \square 10^{12} \square$ \\
4 & $\square 0.0222893 \square 0.0114597 \square$ & 38.3996 & $\square 3.59518 \square 10^{12} \square 1.64443 \square 10^{11} \square$ \\
5 & $0.00108319 \square 0.00751676 \square$ & 161.314 & $\square 6.83748 \square 10^{12} \square 3.88986 \square 10^{11} \square$ \\
6 & $0.00231047 \square 0.00123722 \square$ & 37.2613 & $1.05687 \square 10^{11} \square 3.9168 \square 10^{11} \square$ \\
7 & $0.00099812 \square 0.000258246 \square$ & 80.7445 & $\square 3.48033 \square 10^{12} \square 1.2284 \square 10^{11} \square$ \\
8 & $0.000291682 \square 0.000346561 \square$ & 89.0032 & $6.44379 \square 10^{12} \square 2.32346 \square 10^{11} \square$ \\
9 & $0.0000370438 \square 0.000214621 \square$ & 160.248 & $\square 5.81363 \square 10^{12} \square 5.35141 \square 10^{12} \square$ \\
10 & $\square 0.0000324126 \square 0.000107957 \square$ & 144.703 & $1.17347 \square 10^{11} \square 4.06876 \square 10^{11} \square$
\end{tabular}

Table 4. Fourier Expansion of $\cos ^{2 v} \mathrm{x}$ with complex $v=3.76+2 i$ and its Error Analysis

\begin{tabular}{cccc} 
i & $a_{i}$ & $x^{0}$ & \\
\hline 1 & $0.435053 \square 0.0611116 \square$ & 88.3827 & $\square 1.87976 \square 10^{11} \square 8.81187 \square 10^{11} \square$ \\
2 & $0.242827 \square 0.0373769 \square$ & 2.57109 & $\square 2.68618 \square 10^{13} \square 1.34417 \square 10^{12} \square$ \\
3 & $0.0702689 \square 0.0607666 \square$ & 54.126 & $6.62803 \square 10^{14} \square 3.85721 \square 10^{12} \square$ \\
4 & $\square 0.00236789 \square 0.0246954 \square$ & 51.6004 & $\square 2.45787 \square 10^{12} \square 1.22795 \square 10^{11} \square$ \\
5 & $\square 0.00555658 \square 0.0000654824 \square$ & $\square 71.3137$ & $3.18412 \square 10^{12} \square 2.2592 \square 10^{11} \square$ \\
6 & $0.000436255 \square 0.00123569 \square$ & 52.7387 & $1.56281 \square 10^{12} \square 4.79623 \square 10^{12} \square$ \\
7 & $0.000194994 \square 0.000301375 \square$ & 9.25552 & $\square 1.26582 \square 10^{12} \square 6.31466 \square 10^{12} \square$ \\
8 & $\square 0.000110119 \square 0.0000310005 \square$ & 0.996754 & $\square 3.04423 \square 10^{13} \square 1.42518 \square 10^{12} \square$ \\
9 & $0.0000393337 \square 0.0000131311 \square$ & $\square 70.2482$ & $2.15089 \square 10^{12} \square 1.06969 \square 10^{12} \square$ \\
10 & $\square 0.0000112576 \square 0.0000123446 \square$ & $\square 54.7029$ & $\square 2.2678 \square 10^{12} \square 8.69305 \square 10^{12} \square$
\end{tabular}

\section{TRIGONOMETRIC SERIES REPRESENTATIONS OF $\sin ^{n} \varphi \& \cos ^{n} \varphi$}

\subsection{Formulations}

For $n$ positive integer we deduce from Equations (1) \& (2) after some analysis that:

$$
\begin{aligned}
& \sin ^{n} \varphi=\left\{\begin{array}{l}
\frac{1}{2} A_{0}^{(n)}+\sum_{s=1}^{(n+\delta} A_{s}^{\prime 2} \cos 2 s \varphi \quad ; \quad \text { if } n \equiv \text { even } ; \delta=0 \\
\sum_{s=1}^{n+\delta} A_{s}^{(2)} \sin (2 s-1) \varphi \quad ; \quad \text { if } n \equiv \text { odd } \quad ; \delta=1
\end{array}\right.
\end{aligned}
$$

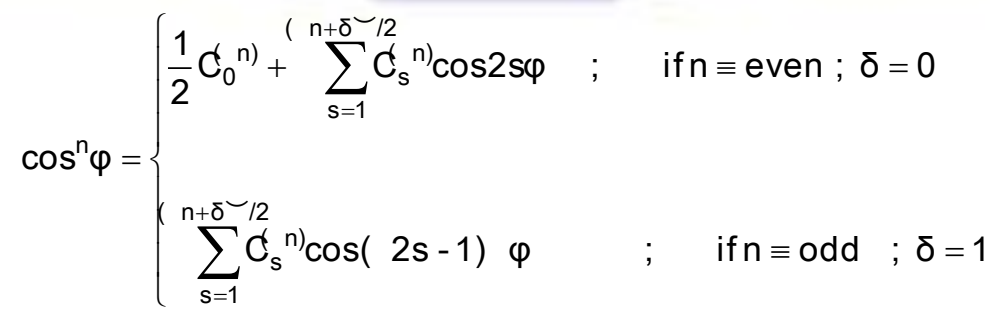

$$
\begin{aligned}
& C_{s}^{m)}=2^{-m+1}\left(\begin{array}{c}
m \\
\frac{m+\delta}{2}-s
\end{array}\right) \quad ; A_{s}^{(m)}=(-1)^{s+\delta} 2^{-m+1}\left(\begin{array}{c}
m \\
\frac{m+\delta}{2}-s
\end{array}\right)
\end{aligned}
$$

We can check the accuracy of the computed values from the conditions that:

- If $\mathrm{n}$ even positive integer, then: 


$$
\frac{1}{2} A_{0}^{(n)}+\sum_{s=1}^{n / 2} A_{s}^{(n)}=0 ; \frac{1}{2} C_{0}^{n)}+\sum_{s=1}^{n / 2} C_{s}^{n)}=1
$$

- If $\mathrm{n}$ odd positive integer, then:

$$
\left.\sum_{s=1}^{(n+1) / 2} 2 s-1\right) A_{s}^{(n)}=0 ; \sum_{s=1}^{(n+1) / 2} d_{s}^{n)}=1
$$

\subsection{Applications}

Tables $5 \& 6$ give the trigonometric series representations of $\sin ^{n} \varphi \& \cos ^{n} \varphi ;$ respectively $; n=2,3, \ldots, 10$.

Table 5. Trigonometric series representations of $\sin ^{\mathrm{n}} \varphi, \mathrm{n}=2,3, \ldots, 10$

$$
\begin{aligned}
& \sin ^{2} \theta=\frac{1}{2}-\frac{1}{2} \cos (2 \theta) \\
& \sin ^{3} \theta=\frac{3 \sin (\theta)}{4}-\frac{1}{4} \sin (3 \theta) \\
& \sin ^{4} \theta=-\frac{1}{2} \cos (2 \theta)+\frac{1}{8} \cos (4 \theta)+\frac{3}{8} \\
& \sin ^{5} \theta=\frac{5 \sin (\theta)}{8}-\frac{5}{16} \sin (3 \theta)+\frac{1}{16} \sin (5 \theta) \\
& \sin ^{6} \theta=-\frac{15}{32} \cos (2 \theta)+\frac{3}{16} \cos (4 \theta)-\frac{1}{32} \cos (6 \theta)+\frac{5}{16} \\
& \sin ^{7} \theta=\frac{35 \sin (\theta)}{64}-\frac{21}{64} \sin (3 \theta)+\frac{7}{64} \sin (5 \theta)-\frac{1}{64} \sin (7 \theta) \\
& \sin ^{8} \theta=-\frac{7}{16} \cos (2 \theta)+\frac{7}{32} \cos (4 \theta)-\frac{1}{16} \cos (6 \theta)+\frac{1}{128} \cos (8 \theta)+\frac{35}{128} \\
& \sin ^{9} \theta=\frac{63 \sin (\theta)}{128}-\frac{21}{64} \sin (3 \theta)+\frac{9}{64} \sin (5 \theta)-\frac{9}{256} \sin (7 \theta)+\frac{1}{256} \sin (9 \theta) \\
& \sin ^{10} \theta=-\frac{105}{256} \cos (2 \theta)+\frac{15}{64} \cos (4 \theta)-\frac{45}{512} \cos (6 \theta)+\frac{5}{256} \cos (8 \theta)-\frac{1}{512} \cos (10 \theta)+\frac{63}{256}
\end{aligned}
$$


Table 6. Trigonometric series representations of $\cos ^{n} \varphi, n=2,3, \ldots, 10$

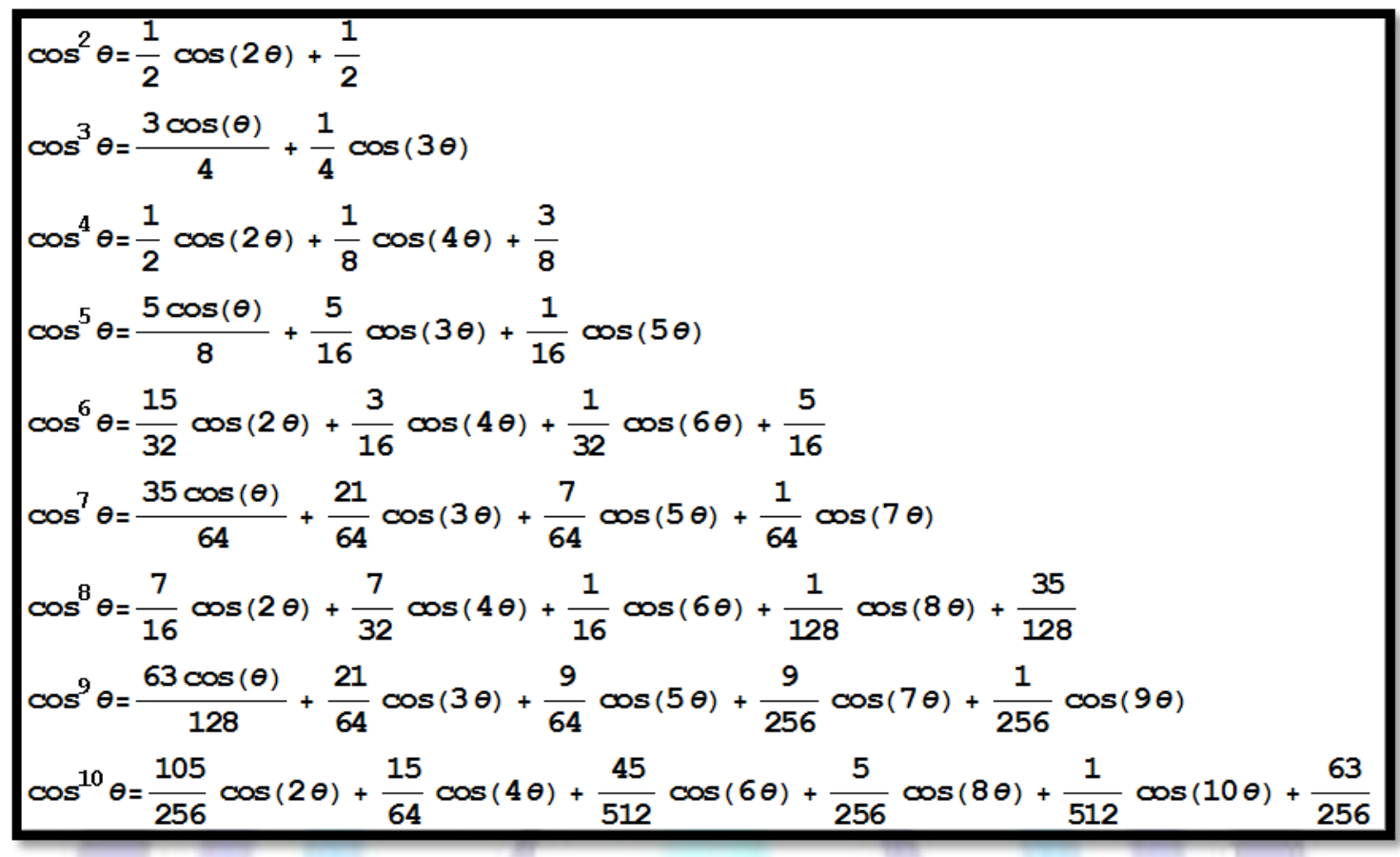

In concluded the present paper, we stress that, Fourier series expansions of powers of sine and cosine functions are established for any possible power-real or complex or positive integer.

Recurrence relations are established to facilities the computations of the coefficients of expansions formulae. Numerical applications for real and complex powers are also included, the accuracy of the computed values are at least of order $10^{-10}$. While the applications for positive integer powers are given as exact analytical expressions.

\section{REFERENCES}

I. Chen Y.M., Meng G. and Liu J.K., 2011, A new method for Fourier series expansions: Applications in rotor-seal systems, Mechanics Research Communications, Vol. 38, Issue 5, pp 399-403.

II. Demircan O., 2005, Basic Functions of the Light Curve Analysis of Eclipsing Variables in the Frequency Domain, Astrophysics and Space Science, Vol.296, Issue 1, pp 209-220.

III. Engheta N., 1999, On Fractional Paradigm and Intermediate Zones in Electromagnetism: II. Cylindrical and Spherical Observations, Microwave and Optical Technology Letters, Vol. 23, Issue 2, pp 100-103.

IV. Jo'dar L., 1990, A separation of the variables method for solving coupled systems of second-order partial differential equations: exact, approximate solutions and error bounds, Journal of Computational and Applied Mathematics, Vol. 31, Issue 2, pp 243-251.

V. Kopal Z., 1975a, Astrophysics and Space Science, Vol.34, 431.

VI. Kopal Z., 1975b, Astrophysics and Space Science, Vol.38, 191.

VII. Kopal, Z., 1979, Language of the Stars, D. Reidel, Dordrecht, The Netherlands.

VIII. Kopal, Z., 1990, Mathematical Theory of Stellar Eclipses, Kluwer Academic, Norwell, MA.

IX. Niarchos P.G., 2005, Contact Binaries: A Study of the Proximity Effects and Gravity Darkening Based on Kopal's Fourier Method, Astrophysics and Space Science, Vol. 296, Issue 1,pp 359-370.

X. Sun Y., Liu S. and Li J., 2009, An improved numerical method for calculations in wave kinematics, Journal of Hydrodynamics, Vol. 21, Issue 6, pp 826-834. 